\title{
Anabases
}

ANABASES Traditions et réceptions de l'Antiquité

Voyageurs et perception des vestiges archéologiques à Sinope au temps de la représentation diplomatique française, sous le Consulat et l'Empire

\section{Claire Barat}

\section{OpenEdition}

\section{Journals}

Édition électronique

URL : http://journals.openedition.org/anabases/1666

DOI : 10.4000/anabases. 1666

ISSN : 2256-9421

\section{Éditeur}

E.R.A.S.M.E.

\section{Édition imprimée}

Date de publication : 1 octobre 2005

Pagination : 163-175

ISSN : 1774-4296

\section{Référence électronique}

Claire Barat, « Voyageurs et perception des vestiges archéologiques à Sinope au temps de la représentation diplomatique française, sous le Consulat et l'Empire », Anabases [En ligne], 2 | 2005, mis en ligne le 01 juillet 2011, consulté le 21 octobre 2019. URL : http://journals.openedition.org/ anabases/1666 ; DOI : 10.4000/anabases.1666 
Anabases 2 (2005), p. 163-175

\section{Voyageurs et perception des vestiges archéologiques à Sinope au temps de la représentation diplomatique française, sous le Consulat et l'Empire}

Claire BARAT

\section{Introduction}

La CITÉ DE SINOPE (aujourd'hui Sinop) se situait sur le littoral sud de la mer Noire, dans le nord de l'actuelle Turquie. Elle est connue depuis l'Antiquité comme une colonie de Milet, fondée au VIIe s. av. J.-C., dans l'endroit le plus propice et le mieux abrité de tout le littoral sud du Pont-Euxin. À l'époque classique, Sinope accueillit un contingent athénien après l'expulsion de ses tyrans par Périclès, en 437 av. J.-C., et vit passer Xénophon, de retour de son Anabase en 401-400 av. J.-C. Sinope s'enorgueillissait aussi d'avoir donné le jour au célèbre philosophe cynique Diogène, autour de 400 av. J.-C. Après le passage d'Alexandre en Asie, Sinope fut une cité indépendante et prospère car durant la période hellénistique, elle n'appartint jamais aux royaumes des Diadoques. Cet âge d'or dura jusqu'à ce qu'elle tombât sous la domination du royaume du Pont en 183 av. J.-C. Sous le règne de Mithridate Eupator, elle fut capitale de son royaume, et à ce titre, ne fut pas saccagée lors de la victoire de Lucullus, en 70 av. J.-C. Au contraire, elle obtint le titre de colonie romaine sous César, en 45 av. J.-C. et fut une cité modèle, intégrée dans la province de Bithynie-Pont. Sinope se maintint dans l'Empire romain puis dans l'Empire byzantin jusqu'à sa prise par les Turcs seldjoukides, en 1214, l'implantation d'un comptoir génois à partir de 1280 et sa prise par les Turcs ottomans en 1461.

Durant l'époque moderne, de nombreux voyageurs sont passés à Sinope ${ }^{1}$, faisant une escale plus ou moins longue et donnant une description de la ville plus ou moins

1 M.E. MARTin, "Some miscellaneous notes on the town and antiquities of Sinop, mainly from travellers' accounts”, Anatolian Studies 48 (1998), p. 175-180. 
détaillée dans leur récit de voyage. Ces témoignages, souvent lus avec attention en raison de descriptions pittoresques et exotiques, n'ont été considérés comme des sources historiques utilisables qu'assez récemment, sous l'impulsion de Louis Robert, qui le premier a montré que les récits de voyageurs permettaient de faire progresser la connaissance archéologique d'une région ${ }^{2}$. Les motifs de voyages, les origines sociales et nationales des voyageurs, ainsi que les itinéraires et les moyens de locomotion empruntés varient selon les voyageurs et les époques ${ }^{3}$. La teneur de leur relation peut aussi varier, ainsi que l'importance accordée aux vestiges archéologiques, c'est-à-dire les vestiges monumentaux visibles à Sinope et les objets tels que les restes sculptés et les inscriptions relatives à l'Antiquité.

À l'époque du Premier Empire, seuls des voyageurs français passent à Sinope et donnent des descriptions de ses vestiges archéologiques. Ces voyages coïncident avec l'existence du consulat de France et l'activité de son consul Pascal Fourcade (18031809). Nous nous proposons d'étudier cette courte période et de déterminer le rôle de Pascal Fourcade dans la connaissance et la perception des vestiges antiques par les voyageurs.

La création d'un consulat de France à Sinope suit la paix signée le 25 juin 1802 entre la France et l'Empire ottoman. Par l'article II du traité, la Sublime Porte consentait à ce que les bâtiments marchands français aient le droit incontestable d'entrer dans la mer Noire et d'y naviguer librement. Pour protéger le commerce français en mer Noire, il fallait des consuls, qui avaient alors le titre de commissaires des relations commerciales. C'est avec une connaissance incertaine de la mer Noire et des produits qui pouvaient y être échangés, et par un choix de villes qui n'était peut-être pas exempt de romantisme, que furent créés, par arrêté du $4^{\mathrm{e}}$ jour complémentaire de l'an X (21 septembre 1802), un consulat ou commissariat général à Sinope, et deux viceconsulats ou sous-commissariats à Héraclée du Pont (Ereğli) et à Trébizonde : ainsi s'ouvrait une période d'exceptionnelle présence française en mer Noire, et surtout à Sinope ${ }^{4}$. Les trois hommes qui allaient défendre les intérêts français en mer Noire étaient Pascal Fourcade à Sinope, Louis Allier à Héraclée et Pierre Dupré à Trébizonde. Les trois nouveaux nommés se réunirent à Toulon où ils devaient s'embarquer : ils appareillèrent le 2 décembre 1802, dans les six bâtiments de la flotte française qui accom-

2 L. Robert, À travers l'Asie Mineure. Poètes et prosateurs, monnaies grecques, voyageurs et géographes, Paris, 1980, p. 1-4.

3 B. Remy, "Les sites côtiers du Pont Euxin, de Terme (Themyskira) à Trabzon (Trapézonte) dans l'Antiquité. II. Les récits de voyageurs", in Ier Colloque international sur la mer Noire, Samsun 1988, Samsun, 1990, p. 562-583.

4 H. Deherain, "Les premiers consuls de France sur la côte septentrionale de l'Anatolie", Revue de l'histoire des colonies françaises 17 (1924), p. 301-380 ; F. BILICI, "La France et la mer Noire sous le Consulat et l'Empire : 'la porte du harem ouverte", in W. ARBID, S. Kançal, J.-D. Mizhrai et S. Saul (dir.), Méditerranée, Moyen-Orient : deux siècles de relations internationales. Recherches en hommage à Jacques Thobie, Paris, 2003, p. 55-92. 
pagnèrent avec éclat le général Brune, ambassadeur de la République auprès de la Porte et mouillèrent devant Constantinople le 6 janvier 1803. Ils durent patienter plusieurs mois à Constantinople avant d'entrer dans leur poste respectif. Pierre Dupré, allant rejoindre son poste à Trébizonde, arriva à Sinope avant Pascal Fourcade.

\section{La visite de Pierre Dupré, consul de Trébizonde}

Pierre Dupré partit de Constantinople à destination de Trébizonde le sixième jour complémentaire de l'an XI (23 septembre 1803) ${ }^{5}$. À la différence des deux autres consuls de la mer Noire, Allier et Fourcade, Dupré n'était pas un lettré : il s'agissait d'un ancien commerçant, qui avait déjà passé treize ans en Orient, après avoir quitté la France pour aller exploiter en Albanie des bois destinés à l'arsenal maritime de Toulon. Il avait été agent commercial en Grèce avant d'être rattaché à la légation française à Constantinople. Le 7 vendémiaire, Pierre Dupré mouilla dans le port de Sinope où se produisit un quiproquo : le consul de Russie, croyant à l'arrivée de Pascal Fourcade, avait envoyé son canot et Pierre Dupré dut indiquer qu'il n'était que le vice-consul de Trébizonde. Après s'être installé et avoir présenté ses compliments au consul de Russie, Pierre Dupré alla parcourir la ville. Il s'attacha d'abord à décrire les murailles de Sinope et à en déterminer l'origine, tout en mentionnant les inscriptions grecques :

"Le château est situé vers le N. Il a été bâti presque tout par les Turcs ; il n’y a que les fondements qui ont été posés par les Génois. Le fort regardant le N.O. a été bâti par ce peuple, mais réparé par les Turcs. Il y a des fondements en entier qui se sont écroulés, et qui ont été rebâtis par ces derniers. Nous vîmes sur une porte du fort une inscription grecque, mais la hauteur nous empêcha de pouvoir la lire. C'est ce qui nous arriva à une autre inscription semblable qui est sur la porte du château, mais qui paroit avoir été mise par les Turcs pour remplir un vuide ${ }^{6}$."

Il visita ensuite dans la citadelle le cachot où dix Français avaient été emprisonnés en représailles à l'expédition d'Égypte, admira deux lions sculptés qu'il jugea d'époque génoise. Il décrivit ensuite la cité en donnant des indications sur la topographie et sur le port. Il fit une remarque peut-être tirée de sources anciennes : "On prétend qu'il y avait autrefois à l'extrémité du fort un canal sur lequel étoit un pont et où passoient les barques et petits navires ${ }^{7}$. " Pierre Dupré n'étant pas pétri de culture classique, il peut difficilement dater les monuments qu'il voit : "L’après dîner, picqués par la curiosité,

5 H. CORDIER, "Voyage de Pierre Dupré de Constantinople à Trébizonde (1803)", Bulletin de la section de géographie 32 (1917), p. 256-264.

6 Cordier, "Voyage de Pierre Dupré,, p. 260-261.

7 Cordier, "Voyage de Pierre Dupré", p. 261. 
nous grimpâmes sur la montagne, et nous vîmes les fondements seulement d'un fort, dans une position admirable, et de forme triangulaire. Un angle regarde Ak-Liman, port situé de l'autre côté de la presqu'isle ; le second, au Sud, bat la ville et le château ; le troisième donne sur l'entrée du port. Il y a auprès de ce fort une maison quarrée surmontée d'un dôme, il paroît que c'était autrefois une ancienne chapelle. Cette enceinte peut avoir $12 \mathrm{p}^{\mathrm{ds}}$ de côté 8 », comme cela se remarque dans ce dernier passage : "On trouve dans la ville de Sinope beaucoup de fontaines avec des inscriptions grecques; ce qui me fait croire qu'elles ne doivent pas être anciennes, c'est qu'elles n'ont rien d'extraordinaire ${ }^{9}$."

Pierre Dupré ne fut pas très sensible aux vestiges archéologiques de Sinope, bien qu'il en notât l'essentiel (la muraille, les inscriptions, les vestiges de la péninsule), mais après tout, il ne resta que cinq jours à Sinope. C'est Pascal Fourcade, en poste à Sinope pendant près de six ans, qui va avoir tout le loisir d'étudier vestiges, inscriptions et monnaies.

\section{Pascal Fourcade, un consul archéologue à Sinope}

C'est à l'été 1803 que Pascal Fourcade réussit à obtenir toutes les autorisations et pièces nécessaires à l'exercice de ses fonctions : il passa donc ensuite de Constantinople à Sinope, sur un bâtiment turc battant pavillon français. Il était né à Pau en 1769, et s'était rendu à Paris après avoir achevé ses études. Là, il s'enflamma pour les idées révolutionnaires et prit la tête de manifestations patriotiques. Il fut nommé consul à SaintJean-d'Acre en 1795, mais il ne remplit pas sa charge, car il dut se rendre à La Canée pour y être consul de France. C'est là qu'il fut emprisonné lors de l'Expédition d'Égypte de Bonaparte en 1798 ; il y resta treize mois, avec sa femme et son premier enfant. Il fut ensuite transféré à Constantinople, toujours pour y être emprisonné. Une fois la paix signée, en 1802, il fut libéré et revint en France, pour être renvoyé en Turquie en tant que consul de France à Sinope ${ }^{10}$. L'homme qui arrive à Sinope en 1803 s'installe dans la seule base navale turque de la côte anatolienne à cette époque. Sinope est alors une ville d'à peu prés 10000 habitants, essentiellement des Turcs et des Grecs, gouvernée par un mutesellim (gouverneur délégué) au nom du pacha de la province de Trébizonde, Tayyar Mahmud ${ }^{11}$.

La première tâche de Fourcade à Sinope fut de se concilier ce pacha, dont dépendait Sinope. Il eut aussi à se défendre contre les menées du consul de Russie, qui voyait

8 Cordier, "Voyage de Pierre Dupré", p. 261.

9 Cordier, "Voyage de Pierre Dupré", p. 262.

10 F. Cumont, "Pascal Fourcade, explorateur de l'Asie Mineure", Comptes rendus de l'Académie des inscriptions et belles lettres (1922), p. 308-317.

11 BilicI, "La France et la mer Noire", p. 62. 
d'un mauvais œil l'arrivée d'un collègue français. Durant l'année 1804, Fourcade s'appliqua à établir de bonnes relations avec le pacha de Trébizonde, afin de le convaincre de l'intérêt qu'il avait à favoriser le développement du commerce français dans ses États. Malheureusement pour Pascal Fourcade, la puissance de son protecteur ottoman fut éphémère, puisqu'en 1805 il était en guerre, fut vaincu et s'enfuit, laissant l'insécurité à nouveau s'installer dans la région.

En 1803, la reprise de la guerre contre l'Angleterre avait à peu près interrompu le trafic français dans la mer Noire. Avec la disparition de Tayyar Mahmud, cet événement jetait Pascal Fourcade dans une inaction forcée, qu'il mit à profit pour explorer la région, d'un point de vue géographique, mais aussi archéologique.

C'est ainsi qu'il fit trois voyages dans le courant de l'année 1806, et rédigea de nombreux mémoires à leur propos ${ }^{12}$. Pendant l'hiver et le début du printemps, il se rendit au sud de Sinope, remontant la vallée du Kizıl Irmak (l'Halys des Anciens), puis celle du Gok Irmak ('Amnias des Anciens), pour arriver à Kastamonu. À la suite de ce voyage, il rédigea le Mémoire sur Pompeïopolis ou Tasch Kouprou avec quelques remarques sur Tovata ou Voyavat, qui fut lu à la troisième Classe de l'Institut en 1811. Fourcade recueillit également des renseignements sur Gangra, qui fut la résidence de Déjotaros, roi de Galatie et rédigea un Mémoire sur Castambol ou Castamouni, qui fut lu par Barbié du Bocage, géographe du ministère, à la première et à la troisième Classe de l'Institut en 1811. Enfin, à la suite de ce voyage, il dressa une Carte de la Paphlagonie, qui resta manuscrite ${ }^{13}$, mais qui fut remise à Barbié du Bocage pour être utilisée par lui 14 . Pascal Fourcade fit un second voyage dans la région. Il profita d'une cure qu'il devait faire aux eaux thermales de Kavsa pour visiter les principales localités de la province d'Amasya, à savoir Bafra, Vezir Köprü, Kavsa, Ladik, Mersivan et Amasya et rédiger des mémoires qui se trouvent soit dans la correspondance consulaire, soit dans les Mémoires et documents des archives du ministère des Affaires Étrangères. Enfin, de retour de Kavsa, Fourcade fit un voyage à Inebolu, l'Ionopolis des Anciens en octobre 1806, afin d'accompagner Amédée Jaubert, de retour de mission auprès du Shah de Perse.

12 P. FourCADE, "Mémoire sur Pompeiopolis ou Tasch-Kouprou avec quelques remarques sur Tovata ou Voyavat lu à la troisième classe de l'Institut", Annales des voyages, de la géographie et de l'histoire 14 (1811), p. 30-58 ; MALTE BRUN, "Nouveaux voyages dans l'Asie Mineure, par un consul général de France", Annales des voyages, de la géographie et de l'histoire 13 (1811), p. 408-409 ; DEHERAIN, "Les premiers consuls", p. 323-341.

13 DeHERAIN, "Les premiers consuls", p. 326 et 347 ; carte conservée à la BnF, section des Cartes et Plans, sous le nom de "Carte d'une partie du pont galatique et de la Paphlagonie par M. Fourcade aîné, commissaire général des relations de France et consul général de S.M. l'empereur Napoléon à Sinope, 1806 ».

14 Cumont, "Pascal Fourcade", p. 313 citant la lettre du ministre à Fourcade, 7 août 1807 (carton de Sinope). 
Si Pascal Fourcade montra son intérêt pour la région où ses fonctions l'avaient conduit, il s'intéressa aussi énormément à la cité de Sinope. Nous savons qu'il rédigea un mémoire, De la situation politique et militaire de Sinope, du 12 février 1807 ${ }^{15}$, mais surtout un Mémoire sur Sinope dont l'existence est attestée par Malte Brun qui en donne une brève notice en 1811 : "Le troisième mémoire de M. F.....e, qui est plutôt un ouvrage qu'un mémoire, roule sur les antiquités de Sinope; il décrit cette célèbre ville dans le plus grand détail, et il en éclaircit l'histoire par les médailles dont il a découvert ou recueilli un très grand nombre. Le grand Mithridate calomnié par les historiens romains, a été bien apprécié par notre voyageur, qui a retrouvé partout et jusque dans le système d'unité monétaire établi dans ses états, des traces manifestes de sa haute politique et de sa magnificence royale ${ }^{16}$. " Nous savons que Fourcade avait mentionné sa somme de travail dans une lettre à Talleyrand du 15 avril $1807{ }^{17}$. Le journal de Fourcade a été conservé aux archives du ministère des Affaires Étrangères ${ }^{18}$, mais il n'en est pas de même de son mémoire sur Sinope : entre 1811, époque où Malte Brun eut ce mémoire sous les yeux, et 1922, date où Franz Cumont écrivit son article, ce mémoire avait disparu et F. Cumont, qui fit des recherches, ne le trouva ni aux Archives de l'Institut, ni aux archives Nationales, ni à la Bibliothèque Nationale, ni aux archives Diplomatiques. Il ne put qu'émettre des hypothèses, à savoir que les manuscrits de Fourcade non retrouvés auraient pu être conservés par Barbié du Bocage, à qui ils avaient été confiés, ou bien se trouveraient en la possession d'un descendant de Pascal Fourcade ${ }^{19}$. Il est donc tout à fait regrettable que ce manuscrit ait disparu car il constituait la première œuvre dédiée uniquement à l'archéologie de Sinope, à une époque où les savants français s'enthousiasmaient pour l'archéologie de l'Égypte. Nous savons que deux plans avaient été exécutés pour illustrer ce travail, intitulés "Plans des bains du gimnase de l'ancienne Sinope levé en pluviôse de l'an 12 par J.-C. Escalon " et "Citernes antiques, réservoirs d'eau pour le Gymnase de Sinope " et qu'ils étaient conservés dans la correspondance consulaire, carton Sinope ${ }^{20}$.

Si nous n'avons malheureusement pas pu retrouver le mémoire de Pascal Fourcade sur Sinope, nous pouvons glaner quelques informations sur sa connaissance archéologique du lieu en consultant ses autres écrits. Par exemple, dans son mémoire sur Pompeiopolis, le seul publié, il fait quelques remarques sur une inscription vue à Sinope :

15 Cité par Deherain, “Les premiers consuls”, p. 347, n.1, dans la liste des Mémoires écrits par Fourcade.

16 MAlte Brun, "Nouveaux voyages", p. 408.

17 AMAE, carton de Sinope, pièce n ${ }^{\circ} 14$, citée par DEHERAIN, "Les premiers consuls", p. 345346.

18 AMAE, carton de Sinope, pièce $\mathrm{n}^{\circ} 34$.

19 CumOnt, "Pascal Fourcade", p. 316-317.

20 Deherain, "Les premiers consuls", p. 342 et 347, n.1. 
" J'ai trouvé le même titre de Pontarque dans les ruines de l'amphithéâtre de Sinope. Il paroît qu'avant la nouvelle division provinciale adoptée par Hadrien, les villes fédérées du Pont célébroient des jeux publics en l'honneur de Sérapis, d'Isis, de Proserpine et de Mars, divinités protectrices de peuples de la mer Noire ; toutes ces villes, à l'exemple de la province asiatique, de la Cilicie, de la Galatie, avoient obtenu sans doute la faveur d'élire un président annuel de jeux et des spectacles que les habitants du Pont célébroient en commun. Le Pontarque de Sinope avoit mérité la reconnaissance de ses compatriotes par des combats de gladiateurs et de taureaux qu'il avoit donné à ses frais et avec la plus grande magnificence. Mais nous en parlerons avec quelque détail quand nous décrirons les ruines de Sinope ${ }^{21}$."

Cette inscription sinopéenne fut ensuite retrouvée, estampée et publiée dans le $C I G^{22}$ avec la référence à Fourcade.

Dans le mémoire Bains et pays de Kavza. Thermes, Pascal Fourcade fait des remarques d'ordre architectural :

"Avec des pierres chargées d'inscriptions grecques ou latines très mal conservées, les murailles de tous ces monuments offrent des briques dont la forme, la couleur et les dimensions sont les mêmes que celles des briques du gymnase de Sinope et du Palais de Mithridate à Samsoun (Amisus Eupatoria) ${ }^{23}$."

Outre ses qualités d'épigraphiste, d'archéologue et d'historien, Pascal Fourcade était numismate : une lettre adressée à Hermand, du 25 prairial de l'an XIII (14 juin 1805) le montre dans son rôle de collectionneur de médailles 24.

Ces quelques remarques montrent l'intérêt que portait le consul de France aux antiquités de Sinope, qu'il s'agisse des monuments, des éléments d'architecture, des inscriptions ou des monnaies. Ce goût pour l'Antiquité le conduisit à rédiger un mémoire sur les monuments antiques de Sinope, malheureusement perdu à ce jour, et qui devait donner en détail ses remarques et ses théories sur ses monuments. Pour pallier cette perte, nous disposons néanmoins des récits de trois voyageurs français, diplomates comme Fourcade, et reçus par lui à Sinope. Nous allons voir comment à travers leurs récits les idées de Pascal Fourcade sont perceptibles.

\section{Pascal Fourcade accueille les voyageurs français à Sinope}

L'ambassadeur de France en Perse, Pierre Amédée Jaubert, fut de passage à Sinope à l'automne 1806, de retour d'une mission pénible. Il était très affaibli et fut accueilli par

\footnotetext{
21 FourCaDE, "Mémoire", p. 38.

$22 \quad C I G 4157=I K(64)$ Sinope, 103.

23 AMAE, Turquie, Mémoires et documents, t. 30, 1741-1808, fo 401-406 ; DEHERAIN, "Les premiers consuls", p. 335.

24 Deherain, "Les premiers consuls", p. 343-344.
} 
Fourcade, dont il sollicita l'aide pour rejoindre Constantinople. Son état de santé ne lui permit pas de visiter la cité, mais il consigna quelques remarques à son sujet dans son récit de voyage :

"La ville de Sinope, dominée du côté du nord par une montage sur laquelle divers auteurs placent le tombeau de Mithridate Eupator, est située sur la partie la plus étroite d'une presqu'île de trois lieues de tour qu'un isthme sablonneux sépare du continent, son enceinte à peu près carrée est formée de vieilles murailles flanquées de tours qui suffiraient à peine, en cas d'attaque, pour la mettre à l'abri d'un coup de main ${ }^{25}$. »

Même si ces remarques sont rapides, la mention de l'emplacement du tombeau de Mithridate Eupator permet de supposer qu'il a eu une conversation avec Fourcade, qui lui a sûrement narré l'histoire de Sinope et ses propres interprétations de la topographie des lieux.

C'est avec le voyageur suivant, Adrien Dupré, que nous trouvons une description de qualité des vestiges archéologiques de Sinope. Adrien, fils de Pierre Dupré, viceconsul de France à Trébizonde, avait accompagné son père dans son poste et fut par la suite lui aussi diplomate, puisqu'il fut consul à Salonique. Il fut l'objet d'un scandale à Trébizonde car il avait introduit des femmes dans sa maison ${ }^{26}$. C'est quasiment en tant que voisin qu'il se rendit à Sinope durant son séjour auprès de son père à Trébizonde. Adrien Dupré commence sa description en donnant des informations d'ordre topographique, appuyées sur une bonne connaissance des textes de Strabon. Il décrit ensuite la péninsule : «Cette presqu'île est montagneuse, et, dans sa partie la plus élevée, se trouve un petit lac d'excellente eau douce, qui alimente les nombreuses fontaines de la ville au moyen de canaux souterrains construits par les anciens Grecs. Ce lac est peut-être celui de Korokondames, qui, suivant Strabon, avoit pris son nom du bourg ainsi appelé, et qui se trouvait à dix stades de la mer. Les fontaines, surmontées de quelques inscriptions grecques , semblent appartenir au Bas-Empire ${ }^{27}$."

Son exploration de Sinope le conduit à émettre des remarques très intéressantes concernant les murailles, les inscriptions et les reliefs de la ville :

" [La ville de Sinope] est entourée de murailles à doubles remparts, défendues par des tours, la plupart triangulaires et pentagones, ne présentant qu'un seul angle. Sur une des tours, près de la mer, est une inscription grecque, au milieu de laquelle est une croix. Cet emblème me fit juger que ces fortifications ne datoient que de l'époque du Bas-Empire. À une grande hauteur, et sur le même côté de cette tour, est une niche, où l'on voit une tête antique en marbre mutilé. La chevelure et le col sont encore bien conservés. Cette

25 P.A. Jaubert, Voyage en Arménie et en Perse, fait pendant les années 1805 et 1806, Paris, 1821, p. 394.

26 DEHERAIN, "Les premiers consuls", p. 373.

27 A. Dupré, "Description de quelques villes et ports de la mer Noire", in Chevalier GAMBA, Voyage dans la Russie méridionale, Paris, 1826, p. 435-436. 
sculpture m'a paru appartenir aux beaux temps de l'art, et aux plus anciennes époques de la Grèce. Elle aura sans doute été placée là par quelque main barbare, qui, en bâtissant, l'aura regardée comme une pierre ordinaire, et propre à remplir son but. Je pourrois en dire autant d'un bas-relief de quinze pouces de haut sur vingt-quatre de large, que j'ai vu sur un mur au bord de la mer. Il est encore bien conservé, et représente un homme nu, à demi-couché sur un lit, une coupe à la main, en face duquel est une figure tenant aussi une coupe, un peu plus loin se trouve nu, les mains posées sur un stade.

Il paroît que l'ancienne Sinope occupoit l'espace compris entre la forteresse dont nous allons parler, et la montagne qui la borde à l'est, c'est-à-dire qu'elle n'étoit pas sur l'isthme, mais bien sur la presqu'île. On ne voit plus aucun de ces murs dont parle Strabon. On reconnoît dans ceux qui existent l'architecture européenne, ou du moins celle des derniers empereurs Grecs. Le château, situé vers le nord, domine la ville ; il a été bâti par les Turcs, les fondements seuls paroissent plus anciens. La partie des fossés a été construite avec des débris de temples et de palais : partout on y voit des colonnes, des architraves. Nul doute que les restes de ce gymnase et de ces superbes portiques, que cite Strabon dans la relation de Sinope, n'aient servi à la construction de ce château. Le grand nombre de colonnes, de tronçons qu'on trouve dans les cimetières, les masses du palais de Mithridate, bâtis en belles briques d'une dimension triple au moins des notres, les restes du superbe gymnase dont parle Strabon, l'étendue et la distribution intérieure de ces vastes édifices, dont on peut se faire une idée par les débris qui existent encore, attestent de l'ancienne grandeur de Sinope, et confirment la relation de ce savant géographe.

Le château forme la presqu'île du côté de la terre ; ses murailles et celles de la ville sont battues des deux côtés par la mer, et, dans sa plus petite largeur, la langue de terre qui unit la presqu'île au port, est d'environ huit cents pas (de deux pieds et demi). On prétend qu'autrefois il y avait à l'extrémité du fort un canal, sur lequel étoit un pont, et où passoient les barques et les petits navires, mais il n'en est question dans aucun auteur ancien. Strabon parle seulement des arsenaux maritimes de la ville et de ses deux ports placés des deux côtés de l'isthme ${ }^{28}$."

Adrien Dupré a bien noté l'abondance des éléments d'architecture présents dans les murailles et dans les cimetières de Sinope : il y a vu des inscriptions grecques et un buste, de même que le bas-relief représentant un homme couché. Adrien Dupré connaît bien le texte de Strabon ${ }^{29}$ et fait habilement la relation entre les restes de ce que l'on appelle le Palais de Mithridate et le gymnase. Il a des connaissances historiques et architecturales puisqu'il propose des datations des vestiges qu'il rencontre (BasEmpire, époque byzantine). Nous pouvons supposer qu'il connaissait le travail de l'as-

28 A. Dupré, "Description”, p. 436-438.

29 Strabon, Géographie, XII, 3, 11. 
tronome Joseph Beauchamp, passé à Sinope en 1797 et qui avait observé les mêmes inscriptions et le même buste ${ }^{30}$, ou bien qu'il avait bénéficié d'une visite guidée de la part de Pascal Fourcade. Nous énonçons cette hypothèse car nous allons voir que les éléments descriptifs du récit d'Adrien Dupré coïncident de manière quasiment parfaite avec la description du voyageur suivant, J.M. Tancoigne.

J.M. Tancoigne se rendit à Sinope de retour d'un voyage diplomatique en Perse, à la suite du général Gardane, chargé en 1807 d'une ambassade auprès du Shah de Perse afin d'entretenir l'inimitié entre Persans et Russes, d'offrir un conseil militaire à la Perse et de préparer une expédition éventuelle vers l'Inde ${ }^{31}$. Il y participait en tant qu'élèveinterprète et fut plus tard interprète et chancelier du consulat de La Canée. Il ne participa pas à l'intégralité de la mission car il faillit être frappé de cécité alors qu'il se trouvait à Téhéran, ce qui entraîna son retour anticipé à Constantinople 32. C'est lors de ce voyage retour que Tancoigne s'arrêta à Sinope et fut accueilli par Fourcade 33 en août 1808. C'est un homme cultivé qui qualifie Sinope d'antique patrie de Diogène, lieu commun présent chez tous les voyageurs du XIXe s. Il fait tout d'abord quelques remarques d'ordre général sur le site, la population et les cultures de Sinope, qui montrent sa connaissance de l'ancienne Sinope : «Sinope ou Sinub, comment l'appellent les Turcs, est située sur un isthme de peu de largeur, qui sert de communication entre le continent et la presqu'île où se trouvoit l'ancienne ville ${ }^{34}$."

La partie la plus intéressante de son texte concerne la visite qu'il fit de la ville avec Fourcade, qui ressemble fort à celle d'Adrien Dupré :

"J'ai parcouru, avec M. Fourcade, toute la presqu'île : elle est montagneuse et peut avoir quatre ou cinq lieues de circonférence. Dans la partie la plus élevée se trouve un petit lac d'excellente eau douce, qui alimente les nombreuses fontaines de la ville, au moyen de canaux souterrains construits par les anciens Grecs. Suivant leur usage, les Turcs laissent dépérir ces ouvrages utiles; leur avarice et leur apathie les empêcheront toujours de les réparer et de les entretenir, à moins que la disette d'eau ne finisse par les y obliger. Au bas de la montagne nous avons vu les ruines du palais de Mithridate, et celles d'un beau gymnase : nous sommes entrés dans toutes les salles de l'un et de l'autre. On peut encore, dans l'état où sont aujourd'hui ces débris, se faire une idée de l'étendue et de la distribu-

30 J. Beauchamp, "Mémoire géographique et historique du voyage de Trébizonde", in J. MORIER, Voyage en Perse, en Arménie et à Constantinople fait dans les années 1808 et 1809, Paris, 1813, p. 282-285.

31 H. DEHERAIN, Orientalistes et antiquaires: La vie de Pierre Ruffin, orientaliste et diplomate (1742-1824), II, Paris, 1929, p. 46.

32 Deherain, Orientalistes, p. 50-51 : lettre de Jouannin à Ruffin du 24 mai 1808 abordant la question.

33 J.M. Tancoigne, Lettres sur la Perse et la Turquie d'Asie, II, Paris, 1819, p. 207.

34 Tancoigne, Lettres, p. 210. 
tion intérieure de ces grands édifices; les murs bâtis en belles briques d'une dimension triple au moins de celles qu'on fait dans nos contrées, sont encore debout. Il n'y manque que les marbres et la toiture ; les Turcs ont enlevé les premiers pour décorer leurs mosquées.

M. Fourcade l'aîné, très versé dans la science des antiquaires, a fait des recherches trèscurieuses sur cette ville et sur ses monuments. Il pense que l'ancienne Sinope occupoit l'espace compris entre la forteresse moderne et la montagne qui la borne à l'est, c'est-àdire qu'elle n'étoit pas sur l'isthme, mais bien sur la presqu'île. Il pense aussi que le terrain a dû considérablement s'exhausser dans cette partie, par des éboulements de terre qu'entraînent les pluies, et que le forum devoit se trouver en face de la porte de la forteresse, à peu de distance de la mer, du côté du Nord. Il estime avec raison que, sous un gouvernement plus éclairé, on parviendroit facilement, à l'aide de quelques fouilles, à exhumer toute l'ancienne ville et un grand nombre de monuments précieux : ce qui doit donner un grand poids à son opinion, c'est qu'on ne peut pas faire un pas sur la presqu'île, surtout après les pluies, sans trouver quelque débris de l'antiquité. Il a déjà rassemblé une collection aussi nombreuse que bien choisie, et beaucoup de médailles rares que les habitants vendent au poids.

L'intérieur de la ville moderne ou de la forteresse offre également une quantité considérable de ruines antiques. Les remparts sont en grande partie bâtis avec des colonnes de marbre blanc, des bas-reliefs et des statues antiques, entassés confusément par la grossière ignorance des Turcs et qui sont aujourd'hui dans un état déplorable de dépérissement. M. Fourcade m'a fait remarquer près de la porte qui regarde la presqu'île, un de ces basreliefs dont les personnages ont la tête en bas. Il a fait auprès du gouvernement turc plusieurs tentatives pour qu'il lui fût permis de le faire enlever de la muraille, et il en a offert un prix raisonnable; mais tous ses efforts ont été inutiles. Il n'en faudroit peut-être pas davantage pour faire une révolution dans le pays. Les Turcs ne conçoivent pas qu'on puisse attacher de prix à des objets pareils, et la curiosité des Européens à cet égard leur paroît toujours suspecte et fondée sur d'autres motifs que ceux qui les dirigent réellement. Ils s'imaginent qu'ils sont poussés par l'espérance de trouver des trésors ou par une superstition dont il leur seroit difficile à eux-mêmes de rendre compte. Il faudroit être plus versé que je ne le suis, Madame, pour vous donner une description de tout ce que Sinope offre d'intéressant à la curiosité du voyageur éclairé. M. Fourcade, mieux que tout autre, puisqu'il réside sur les lieux depuis près de cinq ans, peut en parler ex professo; je sais qu'il se propose de nous donner un jour des renseignements précieux sur cette ville célèbre et sur son territoire ${ }^{35}$."

Tancoigne a suivi un tour quasi immuable pour visiter Sinope et admirer ses monuments : un départ de la péninsule, avec une visite du "palais de Mithridate " et du " gymnase ", puis en ville, l'observation des murailles, où de nombreux éléments

35 TAnCoIgne, Lettres, p. 213-217. 
d'architecture et même des statues et des bas-reliefs sont remployés. Il termine sa visite par la description d'un bas-relief dont les personnages ont la tête en bas. Il est aussi un bon témoin de l'enthousiasme de Fourcade pour l'archéologie de Sinope, ainsi que de ses théories sur l'emplacement de la cité antique et du forum, et sur la géologie de la péninsule. Peut-être que ces hypothèses se trouvaient développées dans le mémoire rédigé par Pascal Fourcade sur Sinope. Nous voyons aussi, grâce au texte de Tancoigne, un Fourcade très préoccupé par les vestiges archéologiques, mais se heurtant à une fin de non recevoir de la part des autorités turques, qui ne veulent entendre parler ni de fouilles ni d'achats d'antiquités. Enfin, c'est dans le récit de Tancoigne que l'on trouve le ton le plus personnel et le plus vif pour fustiger les habitants turcs de Sinope, accusés de piller ou de laisser dépérir les vestiges antiques. Si les descriptions des vestiges de Sinope semblent être une paraphrase des propos de Fourcade, ces vives remarques peuvent être attribuées à la sensibilité de Tancoigne.

Tancoigne fut le dernier voyageur à bénéficier de la culture et des hypothèses de Fourcade. Ce dernier était dans une situation d'inquiétude extrême depuis une agression dont il avait été victime en 1807. Lui et son chancelier Bernage furent sérieusement blessés par des Lazes, qui s'étaient arrêtés à Sinope à cause du mauvais temps, sur le chemin du retour vers Trébizonde, après avoir massacré quelques ministres et déposé le sultan Selim III à Constantinople ${ }^{36}$. Ils s'en prirent à eux en tant que Français et le mutesellim de Sinope ne fit rien pour intervenir. Dans une telle situation, alors que ses agresseurs n'étaient nullement punis ni même inquiétés, Pascal Fourcade sombra dans le découragement et languit pendant l'année 1808, espérant pouvoir quitter Sinope. Le chancelier Bernage se rendit à Constantinople début 1808 afin de préparer le futur départ de Fourcade et de communiquer à Ruffin, le chargé d'affaire à Constantinople, un ouvrage de Fourcade sur Sinope qu'Henri Dehérain considère comme l'embryon de celui dont Malte Brun eut connaissance par la suite et aujourd'hui perdu ${ }^{37}$. Fourcade eut des difficultés avec les fonctionnaires de Sinope qui ne voulaient pas le laisser partir, et ce fut son épouse, accompagnée de Bernage, qui se chargea d'aller chercher un bâtiment à Constantinople et d'y solliciter un firman de départ. Le 17 février 1809, Fourcade s'enfuit de Sinope avec sa famille à destination de la Crimée.

\section{Conclusion}

Sous le Consulat et l'Empire, tous les voyageurs de passage à Sinope furent français et liés à la diplomatie : Pierre Dupré, vice-consul de France à Trébizonde, était accompa-

36 Deherain, "Les premiers consuls", p. 356-363 ; BiLicI, "La France et la mer Noire", p. 64.

37 Deherain, "Les premiers consuls", p. 364. 
gné de son fils Adrien, qui fit ensuite un voyage à Sinope ; Beaujour et Tancoigne firent étape à Sinope de retour d'une mission en Perse.

Cette fréquentation de la petite cité par ces diplomates français est due à l'existence du consulat de France à Sinope, dirigé par Pascal Fourcade de 1803 à 1809. Il reçut tous les voyageurs et leur fit les honneurs de la ville, leur proposant un tour aussi complet que possible et leur détaillant l'histoire et les antiquités de la cité. À travers les récits de voyageurs, il est possible de dresser un portrait de ce diplomate érudit et enthousiaste, véritable archéologue, épigraphiste et numismate.

Les voyageurs offrent une description des vestiges de Sinope assez stéréotypée : description des murailles de la ville où des éléments d'architecture antique et des inscriptions sont utilisés en remploi, description d'un buste et d'un bas-relief antiques. La péninsule attire les voyageurs qui y observent les vestiges de ce qu'ils identifient comme le gymnase de Sinope et le palais de Mithridate. Les voyageurs tentent de mettre chaque élément observé en relation avec les textes antiques.

Guidée par la passion de Fourcade, la perception des vestiges antiques de Sinope n'est pas neutre et ces derniers se font les porte-paroles des hypothèses archéologiques et des observations sur le terrain du consul. Par leur curiosité, ils peuvent être placés dans la continuité des participants de l'Expédition d'Égypte, dont les travaux étaient publiés à la même époque, préfigurant les grands voyageurs du XIXe s., avides de prospections archéologiques et de constitutions de corpus épigraphiques.

Claire BARAT

Université Michel de Montaigne - Bordeaux 3

Ausonius - Institut de Recherche sur l'Antiquité et le

Moyen Âge

Maison de l'Archéologie

clairebarat33@yahoo.fr 